\title{
Expect the unexpected: a paradoxical effect of cue validity on the orienting of attention
}

\author{
Ashley Jollie $^{1}$ • Jason Ivanoff ${ }^{1} \cdot$ Nicole E. Webb $^{1} \cdot$ Andrew S. Jamieson $^{1}$
}

Published online: 27 June 2016

(C) The Psychonomic Society, Inc. 2016

\begin{abstract}
Predictive central cues generate location-based expectancies, voluntary shifts of attention, and facilitate target processing. Often, location-based expectancies and voluntary attention are confounded in cueing tasks. Here we vary the predictability of central cues to determine whether they can evoke the inhibition of target processing in three go/no-go experiments. In the first experiment, the central cue was uninformative and did not predict the target's location. Importantly, these cues did not seem to affect target processing. In the second experiment, the central cue indicated the most or the least likely location of the target. Surprisingly, both types of cues facilitated target processing at the cued location. In the third experiment, the central cue predicted the most likely location of a no-go target, but it did not provide relevant information pertaining to the location of the go target. Again, the central cue facilitated processing of the go target. These results suggest that efforts to strategically allocate inhibition may be thwarted by the paradoxical monitoring of the cued location. The current findings highlight the need to further explore the relationship between location-based expectancies and spatial attention in cueing tasks.
\end{abstract}

Keywords Attention · Inhibition · Precuing

Portions of this work were presented at the $53^{\text {rd }}$ Annual Meeting of the Psychonomic Society

Electronic supplementary material The online version of this article (doi:10.3758/s13414-016-1164-x) contains supplementary material, which is available to authorized users.

Jason Ivanoff

jason.ivanoff@smu.ca

1 Department of Psychology, Saint Mary's University, Halifax, Nova Scotia, Canada

\section{Introduction}

Probabilistic cues feed into the mental preparation for an impending event in different ways. For instance, in an unfamiliar area at night, a sign for a sharp bend in the road alerts the motorist to an imminent change in the driving terrain. The driver may become more alert, pay closer attention to the road, and slow down knowing that these types of signs are very highly predictive of changes to the road. Nonetheless, information about infrequent events is also useful, and may change the driver's mental state. A “deer crossing” sign, for example, is intended to inform drivers of the possibility (albeit slim) that deer are known to the area and may cross the road. It is, by no means, a certainty that a deer will cross the road when the driver sees the sign. Although the frequency of deer crossing may be incredibly low in these areas, these signs are important as they allow drivers to take heed. Drivers may slow down at night and pay closer attention to their surroundings during the day. Thus, signals that are highly, or even just slightly, predictive of future events generate expectations - mental states that reflect information about possible future events (Summerfield \& Egner, 2009). These expectations may alter attention and future behavior to suit the demands of the environment.

Unexpected peripheral onsets can elicit exogenous attention, an unintentional form of orienting (Posner \& Cohen, 1984; Yantis \& Jonides, 1984). Probabilistic cues, on the other hand, guide endogenous orienting: the strategic and deliberate form of attention (e.g., Egeth \& Yantis, 1997; Posner, 1980, 2014). Not only do probabilistic cues guide the endogenous form of attention, they also generate an expectation about the target's appearance. Expectancies and attention facilitate the acquisition of information (e.g., Summerfield \& Egner, 2009). While conceptually independent, these processes may interact (Klein \& Hansen, 1990). In the standard endogenous Posner cueing task, attention and expectation are perfectly 
confounded. In a seminal paper, Posner, Snyder, and Davidson (1980) presented a warning signal $(+)$ or a numeric cue (1-4) at a central location. The numeric cue predicted, with $79 \%$ validity, the location of a target (i.e., the onset of a lightemitting diode). Posner et al. observed that responses to targets at the valid (or cued) location were faster than those at the invalid (or uncued) locations. This facilitative effect is generally taken as evidence for the orienting of strategic, endogenous attention to a particular location in advance of the target. It is thought that the predictability of the cue encourages the observer to use it to direct attention to a particular location in space.

There is still ongoing debate, and research, on the exact mechanisms of visuospatial attention (e.g., see Carrasco, 2011, for a review). Attention is traditionally construed to be a limited resource: it is seen as a process that selects a small portion of information from a vast array (Broadbent, 1958). Early accounts of attention presumed it acted like a spotlight (Posner, 1980), enhancing some objects/locations within the beam, while unattended objects/locations remain untouched. Yet other accounts hold that the allocation of attentional resources to a particular location may come at the expense of resources allocated elsewhere. The perceptual effects of endogenous orienting are only evident under conditions of high external noise when cues are perfectly predictive (Dosher \& Lu, 2000; Lu \& Dosher, 2000), suggesting one of the functions of attention is to exclude visual noise. Others have suggested that spatial attention may operate by inhibiting objects that are neither attended (Cheal \& Gregory, 1997), nor taskrelevant (Cepeda, Cave, Bichot \& Kim, 1998). Neuroimaging evidence (e.g., Slotnick, Hopfinger, Klein, \& Sutter, 2002; Slotnick, Schwarzbach, \& Yantis, 2003) also suggests that attention enhances visual information within the metaphorical spotlight, while inhibiting visual processing outside of it. Thus, it appears as though the strategic allocation of inhibition typically accompanies attentional selection elsewhere in visually cluttered environments.

Is it possible to actively inhibit processing at a location without selecting or enhancing processing at another (i.e., attended) location? We know of no study that has directly attempted to answer this question. However, consider the pioneering work on thought suppression by Wegner and colleagues (Wegner, Schneider, Carter, \& White, 1987). Wegner et al. asked volunteers to avoid thinking of a white bear during a 5-min period. If the thought of a white bear entered their minds, they were to ring a bell. Although instructed not to think of a white bear, there were many intrusions of "white bear"-related thoughts. Other studies have demonstrated similar failures of inhibitory control during the execution of actions (Wegner, Ansfield, \& Pilloff, 1998). Wegner (1994) has interpreted these failures as resulting from an ironic monitoring process that searches for the intrusion of the unwanted thought. The monitoring process is paradoxical, however, as it tends to continually activate the very representation that is meant to be suppressed.

A number of studies have demonstrated top-down inhibitory effects in central cueing tasks where targets are presented with distractor stimuli (Chao, 2010; Lahav, Makovski, \& Tsal, 2012; Moher \& Egeth, 2012; Munneke, van der Stigchel, \& Theeuwes, 2008; Tsal \& Makovski, 2006). In these studies, cues that signal the location of a distractor facilitate the processing of a target presented elsewhere. Tsal and Makovski (2006) dubbed this phenomenon "an attentional white bear," after the classic Wegner et al. (1987) task. It is thought these cues operate by actively suppressing distractor processing. It is plausible that strategic forms of attentional inhibition only operate on distractors that need to be suppressed in the presence (or potential presence) of targets that need to be processed.

There appears to be little evidence for active suppression in the absence of external noise or distracting stimuli. If expectations can direct attention to a location, perhaps they can also guide inhibition. Few studies have considered the relationship between the spatial expectancy from a probabilistic spatial cue and the ensuing shift of endogenous attention (Summerfield \& Egner, 2009). Manipulating the degree to which a cue accurately predicts a target's location is one approach to studying the interface of an expectancy and attention. A handful of studies have directly compared the attentional facilitation from central cues with low predictability to those with high predictability (Eriksen \& Yeh, 1985; Giordano, McElree, Carrasco, 2009; Riggio \& Kirsner, 1997; Vossel, Thiel, \& Fink, 2006; Yantis \& Jonides, 1990). These studies, using arrow (or arrowlike) cues, have shown that the invalid-valid performance difference is increased with the greater proportion of valid trials, suggesting that attention was possibly more focused at the cued location, or the cues were used more often to shift attention to the cued location. No study, of which we are aware, has found direct evidence of top-down, strategic inhibition-in the absence of attentional facilitation - using this methodological approach.

There are three key limitations to previous work manipulating cue validity on endogenous cueing. First, it has been demonstrated that uninformative central arrow (or arrow-like) cues facilitate target processing (Friesen, Ristic, \& Kingstone, 2004; Ivanoff \& Saoud, 2009; Ristic, Friesen, \& Kingstone, 2002; Ristic \& Kingstone, 2006, 2012). Accordingly, a predictive arrow cue might recruit endogenous and exogenous ${ }^{1}$ forms of attention. A second limitation has been the use of two target locations (e.g., Riggio \& Kirsner, 1997; Vossel et al., 2006). Naturally, this approach pits one location (valid) against

\footnotetext{
${ }^{1}$ Alternatively, predictive arrows may recruit decision-level processes (Ivanoff \& Saoud, 2009) or some other form of attention that is neither endogenous or exogenous (e.g., Ristic \& Kingstone, 2012).
} 
another (invalid), and any RT difference between them may be the result of increasing the likelihood of a target appearing at the valid location and/or decreasing the likelihood of a target appearing at the invalid location. While there are some studies that have used more than two locations (e.g., Eriksen \& Yeh, 1985; Yantis \& Jonides, 1990), these studies have focused on increasing the likelihood of a target appearing at a valid location while generally leaving the other locations equally unlikely to possess the target. Lastly, no study has explored the effect of a purely endogenous cue with predictive validity less than chance (i.e., the cue signals the least likely location of the target). A handful of studies have used counter-predictive cues that set endogenous orienting against exogenous orienting (Driver et al., 1999; Friesen et al., 2004; Posner, Cohen, \& Rafal, 1982; Rafal \& Henik, 1994; Warner, Juola, \& Koshino, 1990). Ristic et al. (2002), for instance, observed that uninformative arrows facilitated target detection. Later, Friesen et al. (2004) discovered that counter-predictive arrows (i.e., the direction opposite to the arrow's point indicated the most likely location of a target) resulted in a late facilitative effect at the expected location (i.e., the location opposite to that indicated by the arrow). While this is an extremely powerful approach used to pit endogenous and exogenous forms of attention against one another, these counter-predictive cues still provide the observer with the target's most probable location. No cueing study, of which we are aware, has examined the effect of a cue that is informative about the location that is unlikely to possess a target while not providing information about a location that is more likely to possess a target.

The goal of the current work is to further explore evidence for top-down, strategic inhibition - in the absence of attentional enhancement- simply by manipulating cue expectancy. To accomplish this goal, we used numeric central cues that avoid symbolic, and potentially automatic, cueing effects. The display was designed to have the appearance of an analog clock face with 12 potential target locations. In all experiments, we used the go/no-go paradigm wherein single keypress responses are to be executed to go targets and withheld to no-go targets. In Experiment 1, the cues were completely uninformative with the intent to establish a baseline measure of cueing effects. We included a manipulation of task-context to assess whether these uninformative numeric cues automatically elicit shifts of attention. The goal of Experiment 2 was to compare cueing effects with two types of probabilistic central cues. We measured cueing effects with positive and negative cue-target contingencies. A positive cue-target contingency (i.e., a "probable" cue) is the standard Posner cueing procedure, wherein the cue indicates the location that is most likely to possess the impending target. A negative cue-target contingency (an "improbable" cue), on the other hand, is one where the cue indicates the least likely location of the target. The other uncued locations are all individually (and equally) more likely to receive the target than the cued location. Importantly, the improbable cues are not truly counter-predictive, as they do not inform the participant where the target will most likely appear. Lastly, in Experiment 3, we explored the idea that inhibition is tied to the processing (and suppression) of responses. In this experiment, cues predicted the most likely location of the no-go targets, but did not provide information on the location of go targets.

\section{Experiment 1: uninformative central cues}

The goal of this experiment was to establish a baselineensuring that any effect of the central cues is not the result of automatic orienting. To accomplish this goal, two groups received the same task under different conditions. One group was told that the numeric cue was uninformative and they could ignore it (uninformative and no context). The other group was told that the numeric cue was uninformative, but they were also informed that the display was created to look like the face of a clock and that the central numbers referred to the hour locations on a clock (uninformative with context).

\section{Methods}

\section{Participants}

Forty-six participants (aged 18-27), recruited from Saint Mary's University, volunteered for course credit. Twentythree were randomly assigned to the uninformative group and 23 to the uninformative with context group.

\section{Apparatus and stimuli}

Superlab Pro (Cedrus, CA) was used to record responses and present stimuli on a MAC mini computer. Participants sat $57 \mathrm{~cm}$ away from an 11" Hewlett Packard computer monitor. The placeholders were $1^{\circ} \times 1^{\circ}$ black boxes presented on a white background (see Figs. 1 and 2 for illustrations). The cues were central digits (01-12) that corresponded to the location of a placeholder, as though they were at the hour indicators of a standard analog clock (e.g., "06" is 6 o'clock; see Fig. 2). All placeholders were $6.3^{\circ}$ away from the center of the screen. A placeholder at the center of the screen also surrounded the cue. The go and no-go targets were $1^{\circ} \times 1^{\circ}$ black-filled squares and black-and-white checkerboard-like squares, respectively. 
Fig. 1 A graphical illustration of trial events in Experiments 1, 2, and 3. The placeholders were presented alone for $900 \mathrm{~ms}$ or $1,000 \mathrm{~ms}$ (randomly determined). The central cue was then presented for $1,200 \mathrm{~ms}$. Following the cue, a go target (black-filled square) or a no-go target (checkerboard) was presented. Go targets were presented until a response was executed. No-go targets were presented for $800 \mathrm{~ms}$ or until an erroneous (false alarm) response was made. Responses (simple key-presses with the index finger of the dominant hand on a standard keyboard) were to be made to go targets and withheld to no-go targets

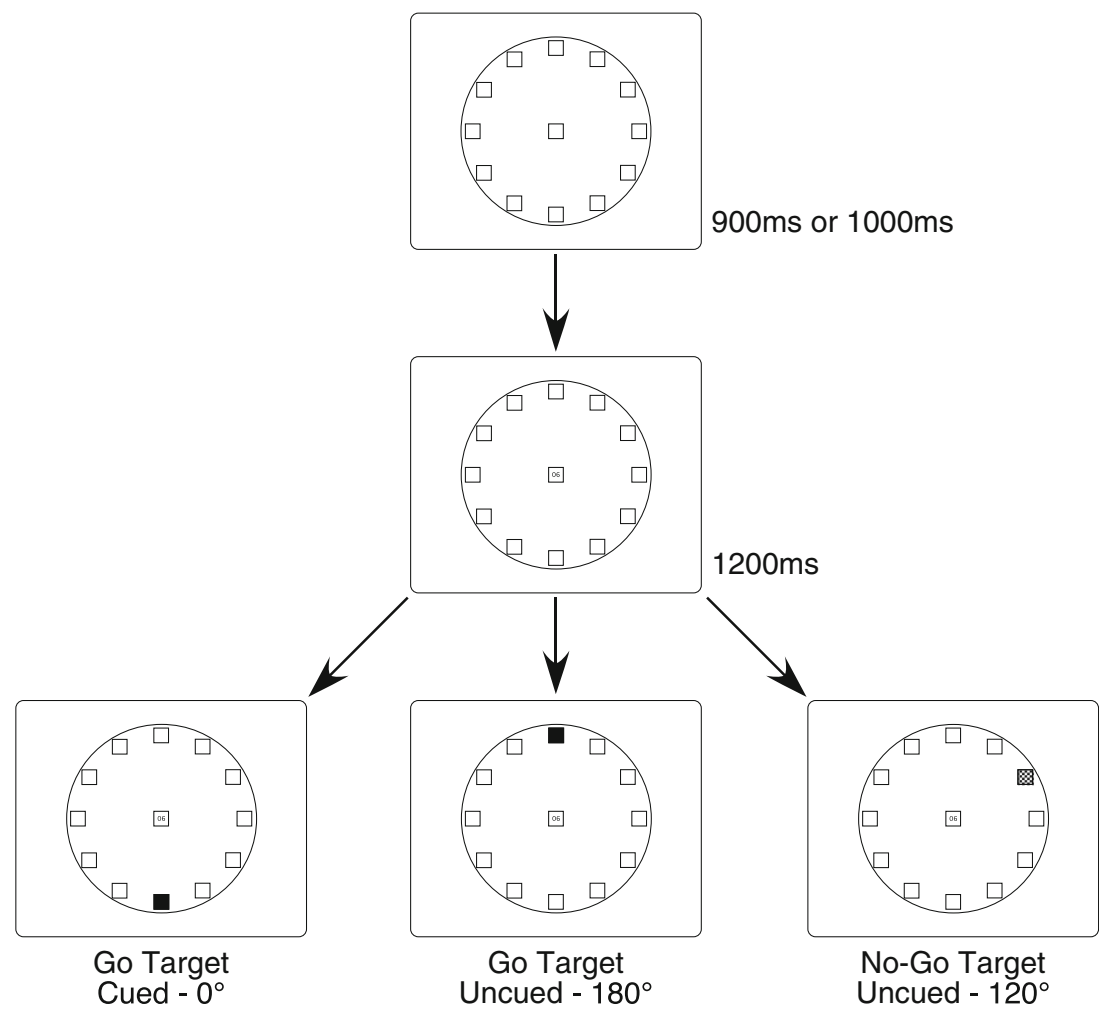

\section{Procedure}

There was no predictive relationship between cues and targets (i.e., the cues were uninformative). The trial procedure is illustrated in Fig. 1. A fixation display, containing the 12 peripheral placeholders (and the one central placeholder) was presented for $900 \mathrm{~ms}$ or 1,000 ms (randomly determined). Following the presentation of the fixation display, the cue appeared in the central placeholder. The cue was presented

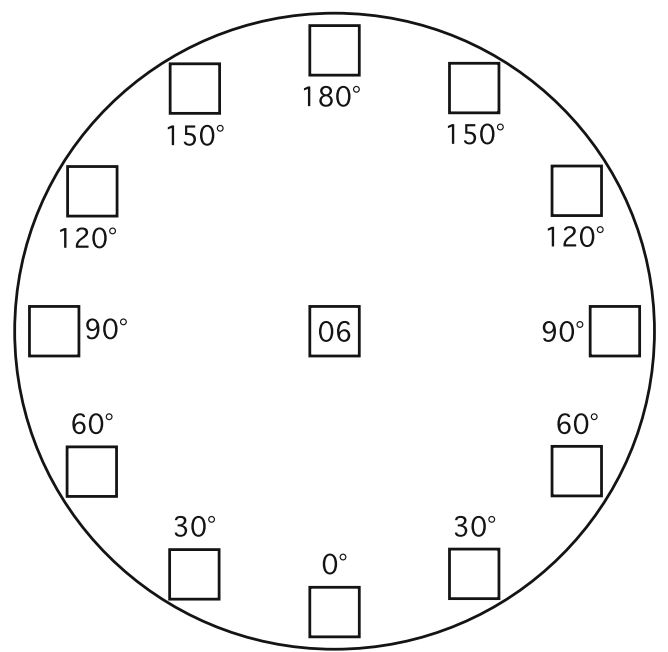

Fig. 2 An illustration of cue-target distance. In this example, the cue (" 06 ") indicates the potential location of a target (go or no-go). A cued target $\left(0^{\circ}\right)$ is one that appears at the location signaled by the cue. Targets appearing at the other locations $\left(30^{\circ}-180^{\circ}\right)$ are uncued. Note that the labels $\left(0^{\circ}-180^{\circ}\right)$ were not actually displayed. See text for more details alone for 1,200 ms. Following this interval, the go or no-go target appeared at one of the 12 locations for 1,000 ms or until a response was made. There were seven possible cue-target distances (i.e., $0^{\circ}, 30^{\circ}, 60^{\circ}, 90^{\circ}, 120^{\circ}, 150^{\circ}$, and $180^{\circ}$ ), as illustrated in Fig. 2. Feedback was presented for $600 \mathrm{~ms}$ whenever the response to the go target was greater than $1 \mathrm{~s}$ (a "Too Slow" message appeared) or whenever any response was made to the no-go target within $800 \mathrm{~ms}$ (a red " $\mathrm{X}$ " appeared at the center). In total, there were 432 go trials and 288 no-go trials. The trial frequencies for each cue and target location are presented in Supplementary Table 1.

There were two groups of participants who received two different sets of instructions. The uninformative group was simply told that the central numbers were uninformative and could be ignored. The uninformative with context group was informed that the display was like a clock face and that the central numbers corresponded to a location on the clock face. They were instructed, however, that the central numbers were not predictive of the target's location.

\section{Results and discussion}

Reaction times (RTs) less than $150 \mathrm{~ms}$ and greater than $900 \mathrm{~ms}$ were excluded from further analysis and were identified as misses. The miss rate was determined by identifying the percentage of go-target trials in which a response did not fall within this RT range. False alarms were responses made to no-go targets. A repeated measures ANOVA on mean RTs, miss rates, and false alarm rates, with instructions 
(uninformative, uninformative with context) as the betweensubject factor and cue-target distance $\left(0^{\circ}, 30^{\circ}, 60^{\circ}, 90^{\circ}, 120^{\circ}\right.$, $150^{\circ}$, and $180^{\circ}$ ) as the within-subject factor, revealed no main effects and no interaction between the factors. ${ }^{2}$ The mean RTs, miss rates, and false alarm rates (i.e., the proportion of erroneous responses to the no-go targets) are presented in Table 1. We estimated the cueing effect by subtracting the mean RT of responses to targets at the cued location from mean RT of all responses to targets at the uncued locations $\left(30^{\circ}, 60^{\circ}, 90^{\circ}\right.$, $120^{\circ}, 150^{\circ}$, and $180^{\circ}$ ) for the uninformative and the uninformative with context groups. Neither differed significantly from zero ( $p$ values $>0.45$ ) and the effect sizes were extremely small (uninformative: Cohen's $d=0.056$; uninformative with context: Cohen's $d=-0.158$ ). See Fig. 3 for a summary of the cueing effects.

The results from Experiment 1 suggest that uninformative, and non-predictive, numeric cues are not processed to the extent that they evoke a shift of attention to the cued location. There was also no evidence that the clock context resulted in an automatic symbolic form of orienting. Thus, we are confident that any orienting effects from these numeric cues are not the result of an automatic form of attentional orienting.

\section{Experiment 2: probable and improbable central cues}

In this experiment we manipulated the predictability of the cues simply by increasing, or decreasing, the frequency of cued (i.e., $0^{\circ}$ ) trials. In the probable condition, the relative frequency of cued trials was increased. In other words, given a cue (e.g., " 06 ") the go and no-go targets were more likely to appear at that peripheral location (i.e., " 06 ") than they were at any other

\footnotetext{
2 There is a long-standing controversy over the use of null hypothesis significance testing in science (e.g., Nickerson, 2000) and there is a possible danger, of course, that we have committed a Type II error. A Bayesian approach may overcome the limitations of null hypothesis significance testing by providing an assessment of the weight of evidence for one model over another (e.g., Rouder, Speckman, Sun, Morey, \& Iverson, 2009; Wagenmakers, 2007; Wagenmakers, Lodewyckx, Kuriyal, \& Grasman, 2010). The JZS Bayes Factor $\left(\mathrm{BF}_{01}\right)$ provides the likelihood of the data under the null $\left(\mathrm{H}_{0}\right)$ versus the alternative $\left(\mathrm{H}_{1}\right)$. Here, we ran a Bayesian repeated measures ANOVA with default priors (Rouder, Morey, Speckman, \& Province, 2012) using JASP software (Love et al. 2016) to complement the analysis. We considered three alternative models to contrast against the null: one that includes a main effect of cue-target distance, another that includes both cue-target distance and instructions (two main effects), and one that includes the two main effects and their interaction. First, the null model was preferred to a model with cue-target distance by a Bayes factor of 274.35. Second, the null model was preferred to a model that includes both main effects $\left(\mathrm{BF}_{01}=344.18\right)$. Lastly, the null model was also preferred to the model that includes both main effects and their interaction $\left(\mathrm{BF}_{01}=1,143.40\right)$. Similar patterns were also observed for the analysis of false alarm and miss rates. Together, these findings convincingly indicate that the data are much more likely to be observed under the null hypothesis, supporting the conclusion that uninformative, central numeric cues are not processed to the degree that they evoke a shift of attention.
}

location (e.g., "09"). It is anticipated that the cues will generate an expectancy and will guide attention to peripheral locations prior to the onset of either a go or no-go target. Responding to cued targets should be faster than responding to uncued targets. In the improbable condition, the relative frequency of cued trials was decreased. In other words, go and no-go targets were unlikely to appear at the cued location. If inhibition can be pre-allocated to locations strategically, then it is expected that responses to cued targets will be slower than responses to uncued targets.

\section{Methods}

\section{Participants}

There were 20 participants in each of the probable (i.e., a positive cue-target contingency) and improbable (negative cue-target contingency) conditions (age range, 18-27). Participants received course credit for their participation.

\section{Apparatus, Stimuli, and Procedure}

The apparatus and stimuli were identical to that used in Experiment 1. The procedure in Experiment 2 was the same as it was in Experiment 1 with the following exceptions. In the probable condition, the participants were informed that the cues would predict the most likely location of the target. Those in the improbable group were instructed that the cues would indicate the least likely location of the target.

Before starting the session, participants had a practice block consisting of 46 trials. During the experimental session, there were ten unscheduled, randomly inserted breaks. In the probable condition there were 504 go, and 312 no-go, trials. The go targets were 3 times more likely to appear at the cued location than any of the other single locations, while the no-go targets were 2 times more likely to appear at the cued location (see Supplementary Table 2 for the trial frequencies). In the improbable condition there were 408 go, and 276 no-go, trials. The go targets were 3 times less likely to appear at the cued location than any other single location. The no-go targets were 2 times less likely to appear at the cued location (see Supplementary Table 3 for the trial frequencies).

\section{Results and discussion}

Errors and RTs less than $150 \mathrm{~ms}$ and greater than $900 \mathrm{~ms}$ were excluded from further analysis. This RT criterion eliminated less than $3 \%$ of all trials in both probable and improbable cue conditions. The average of the remaining RTs for each participant was entered into a repeated measures ANOVA with cue probability (probable or improbable cues) as a betweensubject factor and cue-target distance $\left(0^{\circ}, 30^{\circ}, 60^{\circ}, 90^{\circ}\right.$, $120^{\circ}, 150^{\circ}$, and $180^{\circ}$ ) as the within-subject factor. 
Table 1 Mean RTs (ms), mean false alarm rates $(F A R ; \%)$, and mean miss rates $(M R ; \%)$ as a function of cue-target distance in each experiment for each cue type/instruction
Fig. 3 Mean cueing effects (mean RTs for targets at uncued locations - mean RT for targets at the cued location) in Experiment 1 (uninformative cues and uninformative cues with context), Experiment 2 (improbable and probable cues), and Experiment 3 (no-go probable). The error bars are the standard errors of the difference score; ${ }^{*} p<0.05$

\begin{tabular}{|c|c|c|c|c|c|c|c|c|}
\hline & & \multicolumn{7}{|c|}{ Cue-target distance } \\
\hline & & $0^{\circ}$ & $30^{\circ}$ & $60^{\circ}$ & $90^{\circ}$ & $120^{\circ}$ & $150^{\circ}$ & $180^{\circ}$ \\
\hline \multirow{6}{*}{$\begin{array}{l}\text { Exp. } 1 \\
\text { Uninformative }\end{array}$} & RT & 410 & 412 & 412 & 411 & 411 & 407 & 416 \\
\hline & & $(50)$ & (59) & (53) & (53) & (49) & (51) & $(58)$ \\
\hline & FAR & 2.72 & 2.99 & 2.99 & 2.81 & 2.54 & 3.08 & 2.90 \\
\hline & & $(4.96)$ & $(2.65)$ & $(2.80)$ & $(2.48)$ & $(2.88)$ & (3.19) & (2.93) \\
\hline & MR & 2.29 & 2.60 & 2.05 & 2.17 & 2.72 & 3.14 & 2.66 \\
\hline & & $(3.62)$ & (4.11) & $(2.68)$ & $(3.32)$ & $(4.20)$ & $(4.55)$ & (3.60) \\
\hline \multirow{6}{*}{$\begin{array}{l}\text { Exp. } 1 \\
\text { Uninformative with context }\end{array}$} & RT & 410 & 409 & 408 & 406 & 406 & 411 & 404 \\
\hline & & (46) & $(52)$ & (49) & $(51)$ & (47) & $(50)$ & (48) \\
\hline & FAR & 3.26 & 2.54 & 3.17 & 1.99 & 3.08 & 2.72 & 1.09 \\
\hline & & $(4.34)$ & $(2.81)$ & $(3.38)$ & $(2.63)$ & $(3.71)$ & $(2.03)$ & (1.87) \\
\hline & MR & 2.42 & 1.81 & 1.75 & 2.42 & 1.99 & 2.78 & 2.90 \\
\hline & & $(2.69)$ & $(2.79)$ & $(2.02)$ & $(2.72)$ & $(2.25)$ & $(3.72)$ & (3.70) \\
\hline \multirow{6}{*}{$\begin{array}{l}\text { Exp. } 2 \\
\text { Improbable }\end{array}$} & $\mathrm{RT}$ & 393 & 408 & 405 & 407 & 407 & 409 & 410 \\
\hline & & (43) & (44) & (47) & (53) & (47) & (46) & (53) \\
\hline & FAR & 3.75 & 2.81 & 4.38 & 4.69 & 3.75 & 4.06 & 3.13 \\
\hline & & $(5.72)$ & (3.33) & $(4.53)$ & $(4.27)$ & (3.28) & $(3.97)$ & (4.03) \\
\hline & MR & 4.58 & 1.60 & 2.08 & 1.53 & 2.15 & 2.22 & 1.39 \\
\hline & & (7.39) & (2.64) & $(2.65)$ & $(2.38)$ & (2.18) & $(2.32)$ & (2.63) \\
\hline \multirow{6}{*}{$\begin{array}{l}\text { Exp. } 2 \\
\text { Probable }\end{array}$} & RT & 401 & 410 & 412 & 413 & 416 & 412 & 410 \\
\hline & & (40) & (45) & (43) & (47) & (47) & (49) & (48) \\
\hline & FAR & 7.71 & 4.58 & 5.00 & 4.06 & 4.90 & 5.31 & 5.42 \\
\hline & & $(5.06)$ & $(3.80)$ & $(3.85)$ & (3.91) & $(4.55)$ & $(2.91)$ & (4.50) \\
\hline & MR & 2.92 & 2.85 & 2.50 & 3.13 & 3.13 & 1.67 & 2.92 \\
\hline & & $(3.40)$ & $(3.07)$ & $(2.65)$ & $(2.65)$ & (3.98) & $(1.47)$ & (3.66) \\
\hline \multirow{6}{*}{$\begin{array}{l}\text { Exp. } 3 \\
\text { No-go probable }\end{array}$} & RT & 405 & 410 & 413 & 413 & 415 & 415 & 420 \\
\hline & & (48) & (44) & (47) & (42) & (44) & (43) & (45) \\
\hline & FAR & 1.25 & 0.58 & 0.75 & 0.92 & 0.92 & 1.08 & 0.83 \\
\hline & & $(1.37)$ & (1.13) & (1.18) & $(1.21)$ & (1.91) & (1.71) & (1.70) \\
\hline & MR & 2.00 & 2.83 & 2.58 & 3.08 & 4.08 & 2.50 & 4.83 \\
\hline & & (4.19) & $(3.70)$ & (4.18) & $(3.07)$ & (5.33) & $(4.08)$ & (8.31) \\
\hline
\end{tabular}

Standard deviations are presented in parentheses

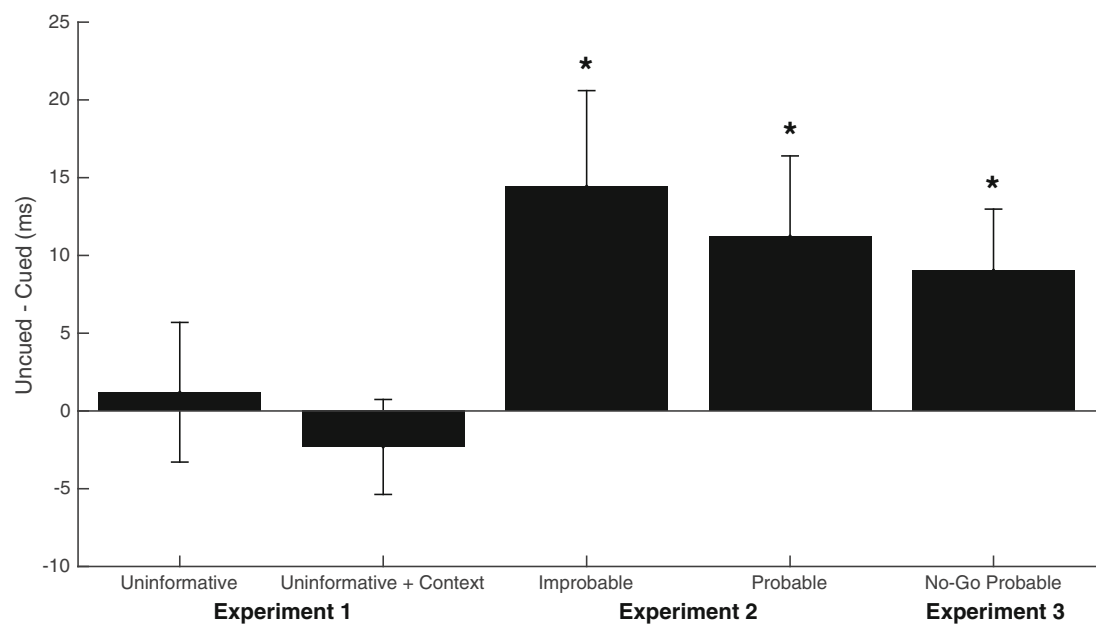


Greenhouse-Geisser corrections were used to correct for violations of sphericity. The analysis of RTs revealed a main effect of cue-target distance $[F(3.18,120.83)=4.52, M S e=$ $403.91, p<0.005]$, but no effect of cue probability $[F(1,38)=$ $0.13, p=0.72$, power $=0.06]$. The interaction between cuetarget distance and cue probability was not significant $[F(3.18$, $120.83)=0.51, p=0.69$, power $=0.16]^{3}{ }^{3}$ The cueing effects both differed significantly from zero [probable: $t(19)=2.19, p$ $<0.05$; improbable: $t(19)=2.35, p<0.05]$, and the effect sizes were numerically larger than they were in Experiment 1 (probable cues: Cohen's $d=0.489$; improbable cues: Cohen's $d=$ $0.526)$. The cue-target distance effect for the probable and improbable cueing conditions is provided in Table 1 and the cueing effect is graphically illustrated in Fig. 3. None of the effects within the analysis of miss or false alarm rates were significant after the sphericity corrections. ${ }^{4}$ Mean RTs, miss rates, and false alarm error rates are found in Table 1.

Not surprisingly, probable cues facilitated target processing. Paradoxically, the improbable cues also facilitated target processing. This latter finding does not support the idea that observers can strategically pre-allocate inhibitory resources to a particular location. It is quite possible that attentional forms of inhibition are contingent on the presence of an intention to selectively attend other locations in (or prior to the presentation of) cluttered visual arrays. However, there is an alternative possibility. Some studies have found putative evidence for strategic inhibition when distractors are cued (e.g., Chao, 2010). Perhaps strategic inhibition is not tied to attentional processing, per se, but to responding. In these studies, distractors are stimuli that should not elicit a response because they are not task-relevant. Accordingly, they are not unlike no-

\footnotetext{
${ }^{3}$ A repeated measures Bayes analysis with default priors (Love et al. 2016; Rouder et al., 2012) was again performed on the RTs. A model that only included the main effects (cueing and cue probability) was preferred over a model that included the interaction by a JZS Bayes Factor of 27.26. Thus, the data provide strong evidence against the hypothesis that cue-target distance and cue probability interact.

${ }^{4}$ Although the interactions between cue probability and cue-target distance were not significant in the analysis of false alarms, we explored the data further because a previous study (Posner, Snyder, \& Davidson, 1980) reported pilot work in which probable cues were used in the context of a go/ no-go task, like the one we report here. In their study, Posner et al. (1980) noted that there was a "strong tendency to react with a false alarm to a visual event occurring in an expected position" and that "it felt as if one were all set to respond when an event occurred in the indicated position" (p. 167). Thus, we performed dedicated $t$-tests comparing the false alarm cueing effects in the probable and improbable conditions. There were more $2.83 \%$ more false alarms to no-go targets at the cued location than there were to no-go targets at uncued locations in the probable condition $[t(19)=$ $3.12, p<0.01]$. The false alarm cueing effect in the improbable condition was not significant $[t(19)=-0.05, p>0.95]$. This finding suggests that although the magnitude of the RT cueing effects does not distinguish between attentional orienting effects in the probable and improbable conditions, it does not necessarily imply that these effects are identical. It is plausible that probabilistic cues affect processing at early and late stages (e.g., Klein \& Hansen, 1990), while improbable cues do not. This idea, however tempting, is speculative and requires further investigation.
}

go targets. Thus, central cues that predict the location of no-go targets (like those that predict the location of distractors) may encourage the voluntary pre-allocation of inhibition. These cues should inhibit the processing of go targets presented at those locations. We test this proposal in Experiment 3.

\section{Experiment 3: probable no-go cues}

In this experiment, we tested the idea that inhibitory processing is tied to responding and not to attention. To accomplish this goal, cues were only informative with respect to the location of the no-go targets. The cues were uninformative with respect to the location of go targets. Accordingly, responding to the cued location should be actively inhibited, as these locations were most likely to have no-go targets.

\section{Methods}

\section{Participants}

Twenty-six undergraduate students participated in Experiment 3 (aged 19-25). Course credit was given as compensation for participation.

\section{Apparatus, stimuli and procedure}

The apparatus and stimuli were identical to Experiment 1 . The procedure was the same with the exception of cue-target frequencies. In the current experiment, the cue was informative with respect to the location of no-go targets. We altered the probability of the go targets and the no-go targets such that no-go targets would appear at the cued location with a greater frequency (see Supplementary Table 4). At the cued location, a go target was 6 times more likely to appear than a no-go target. At the uncued locations, go and no-go targets were equiprobable. In total, there were 408 no-go targets and 288 go targets. Participants were informed that the cue accurately predicted the location of no-go targets, but not the location of the go targets. They were also told that there would be more no-go targets at the cued location than there would be $g o$ targets.

\section{Results and discussion}

RTs less than $150 \mathrm{~ms}$ and greater than $900 \mathrm{~ms}$ were again excluded from further analysis. This criterion eliminated less than $2 \%$ of all trials. One participant made more than $50 \%$ misses in one data cell ( $38 \%$ miss rate overall). The data from this volunteer was removed from further analyses. RTs to go targets were entered into a repeated measures ANOVA with cue-target distance as the only factor. The cue-target distance effect was significant, $F(3.87,92.85)=2.95, M S e=270.3, p<$ 0.05. As seen in Table 1, and Fig. 3, responses to go targets 
were faster to targets at the cued location than they were to go targets at the uncued locations. The cueing effect was significantly different from zero $[t(24)=2.30, p<0.05]$ and its magnitude (Cohen's $d=0.460$ ) was comparable to those in Experiment 2 and much larger than those in Experiment 1. The effects of cue-target distance in the analysis of miss and false alarm rates were not significant.

Cues that predict the location of no-go targets appear to facilitate the processing of go targets. If strategic inhibition were tied to responding, we would have expected slower responding to go targets at the cued location. These results do not support any account wherein observers strategically pre-allocate inhibitory processes to locations in the absence of a set to attend elsewhere.

\section{General discussion}

Experiment 1 convincingly demonstrated that uninformative numeric cues, with or without knowledge of the clock-like context of the display, do not elicit shifts of attention and improve target processing. Importantly, a central cue, accurately predicting the location of a target (Experiment 2, probable cue), does evoke a shift of attention and facilitates target processing. This finding is generally consistent with the vast literature on endogenous cueing effects (e.g., Posner, 1980, 2014). In Experiment 2 (the improbable condition), a central cue that indicated the least likely location of an impending target also facilitated target processing. In Experiment 3, a cue that accurately predicted the location of a no-go target, but was not predictive of the location of a go target, ironically facilitated go target processing. Thus, the current findings do not provide support for an attentional process that can strategically pre-allocate inhibition prior to the onset of a simple target. Rather, these findings implicate an unusual, rather paradoxical, sort of attentional cueing effect that appears to be isolable from a spatial expectancy. We discuss the implications of these findings below.

\section{Is it possible to actively inhibit a location?}

As reviewed earlier, there appears to be good evidence for attentional inhibition in the context of other tasks (e.g., Chao, 2010; Slotnick et al., 2003). In these studies, the inhibition of irrelevant visual information may be accompanied by instructions to attend elsewhere and it is linked to the impending processing of distractors. Thus, the inhibition of a particular location may not be under direct strategic control, but instead may be a byproduct of attentional selection.

Voluntary response inhibition (e.g., Verbruggen \& Logan 2008), distractor inhibition (e.g., Munneke et al., 2008; Tipper, 2001), and the inhibitory aftereffects of overt and exogenous orienting (e.g., Posner \& Cohen, 1984; Taylor \&
Klein, 2000) are not disputed here. Rather, clear evidence of an isolable, and voluntary, form of attentional inhibition is currently lacking. Posner and Cohen (1984; see also Rafal Calabresi, Brennan, \& Sciolto, 1989) failed to observe location-based inhibition following the retraction of a covert endogenous shift of attention. Although Rafal et al. (1989) observed an inhibitory effect following the cancellation of an endogenously prepared saccade (i.e., overt orienting), recent work has failed to replicate this finding (Chica, Klein, Rafal, \& Hopfinger, 2010). Thus, the evidence for strategic forms of isolable, attentional inhibition is not particularly overwhelming.

Other studies appear to directly implicate strategic inhibition without concomitant attentional selection. For instance, in a central cueing task, Chao (2010; Experiment 6) presented observers with two types of trials. On "selection" trials, the goal was to localize a target and ignore a simultaneously presented distractor. A central arrow preceded the target/ distractor array and pointed to one of four locations. The cue pointed to the distractor on $80 \%$ of the trials, and to neither the distractor nor the target on the remaining $20 \%$. Under these conditions, Chao (2010) observed faster responses to targets when the cue pointed to the distractor than when it pointed to neither, suggesting that the cue facilitated target processing by inhibiting the distractor. On "dot" trials (which were less frequent than, and randomly mixed in with, the selection trials), a dot probe would appear at one of the four locations (with equal likelihood) in place of the target/distractor array. Responses to the dot were slower when it was presented at the cued location than at the other locations, suggesting it was inhibited. Our facilitative effect from a probable no-go cue (Experiment 3) seems contradictory to Chao's dot probe results. In Chao's experiments, the cue (an arrow) never pointed to the location of a target, it frequently pointed to the location of a distractor ( $\sim 63.2 \%$ of all trials), and it rarely ( $\sim 5.3 \%$ of all trials) pointed to the location of the dot probe. In contrast, our probable no-go cue identified the location of a no-go target on $\sim 20.6 \%$ of all trials and the location of a go target on $\sim 3.4 \%$ of all trials. Although it is possible that there was little incentive (e.g., Chao, 2010) to actively inhibit the cued location with probabilistic cues in the current study, there was certainly no incentive to actively facilitate cued targets.

Perhaps the strategic inhibition of a location is possible, but it is effortful, subtle or delicate, and therefore difficult to observe. Alternatively, it may be spurious. It is plausible that Chao's (2010) findings (and possibly others) are not the result of strategic inhibition, per se; they may be the result of bottom-up, inhibitory oculomotor processes. Ocuolomotor inhibition of return (Klein \& Hilchey, 2011) is observed whenever a saccade is made and withdrawn from a particular location. It does not have to occur in the presence of peripheral stimulation (e.g., Taylor \& Klein, 2000). If the cue in Chao's study elicited an eye movement to the cued location that was 
withdrawn before the distractor or dot probe appeared, then the evidence for top-down inhibitory processing may be contaminated by a bottom-up ocuolomotor inhibition of return process. Efforts must be made to preclude bottom-up inhibitory processing in any study that purportedly demonstrates the strategic pre-allocation of visuospatial inhibition.

\section{Why do improbable cues, and probable no-go cues, ironically facilitate the processing of the target?}

Strategic mechanisms that attempt to exercise attentional inhibition at a location may be self-defeating because of a monitoring process (e.g., Wegner, 1994). Paradoxically, bringing to mind something that should be inhibited may inadvertently activate the neural pathways for that thought, stimulus, or location. Indeed, simply holding a particular object or feature in working memory can unintentionally guide attention to a location occupied by that same object or feature in a cluttered search array (e.g., Downing, 2000; Olivers, Meijer, Theeuwes, 2006; Soto, Heinke, Humphreys, \& Blanco, 2005). We have found that central, meaningful cues facilitate target processing in simple (i.e., non-cluttered) visual displays. Thus, meaningful central cues may paradoxically evoke a shift of attention, ultimately preventing (or possibly masking) the active inhibition of targets. Uninformative cues (as in Experiment 1) do not appear to engage the monitoring process.

There are a number of subtle methodological and conceptual factors that may limit the generalizability of the paradoxical cueing effects we report in Experiments 2 and 3. First, in all of these experiments, we used a go/no-go task with a go:no-go ratio of $\sim 3: 2$. Ratios of 3:1 are more common and our relatively low go:no-go ratio may have discouraged the automaticity of the go response. ${ }^{5}$ However, an even (or a nearly even) go:no-go ratio may encourage greater perceptual processing of the target, and thereby demand more attentional resources. It remains to be seen whether paradoxical cueing effects occur in perceptually simpler (e.g., detection) tasks. Second, we have used a relatively large number (i.e., 12) of potential target locations, where far fewer (i.e., 2 or 4 ) are more common. Thus, there may be a greater degree of location uncertainty in the present study than there is in other studies with fewer target locations. The effects of paradoxical attention cueing may be contingent on greater location uncertainty. Increasing the number of target locations reduces the overall predictability of the cue: across all experiments, the target was more likely to appear at one of the eleven uncued locations than at the single cued location. Although this ought to have fully discouraged the use of the cues, strangely, the cues still facilitated the processing of the go target. Third, our cues remained present during the presentation of the target display. This procedure is not uncommon and was frequently used in

\footnotetext{
${ }^{5}$ We thank an anonymous reviewer for making this suggestion.
}

early studies of central cueing (e.g., Posner et al., 1980). Strategic inhibition may be impossible while the cue is visible. The presence of the cue may have encouraged a comparison between the cue's specified location with the target's actual location. It seems plausible that this (potentially implicit) matching process may have inadvertently facilitated processing of the go target. Yet, this sort of a comparative process should have facilitated $g o$ target processing in Experiment 1 (and, importantly, it did not). Fourth, we have only explored the paradoxical cueing effect with a single cue-target onset asynchrony (i.e., 1,200 ms). Strategic inhibition may require a longer preparatory interval, as is seen with inhibitory processes stemming from peripheral cues (e.g., Posner \& Cohen, 1984). Lastly, the paradoxical facilitative effects of the cue may be the result of an exogenous, rather than an endogenous, shift of attention. ${ }^{6}$ Extracting the meaning from a central cue may be effortful and under voluntary control, but the ensuing shift of attention may be involuntary. This is, perhaps, akin to what is observed in search tasks while holding an object or feature in working memory (e.g., Downing, 2000; Soto et al., 2005). Future work will need to determine the degree to which paradoxical cueing effects are under voluntary control.

In conclusion, the present findings do not provide support for the strategic inhibition of a location that is isolable from attentional facilitation. This does not imply that strategic forms of inhibition do not exist. However, given that it is difficult to isolate in simple (i.e., not visually cluttered) laboratory tasks suggests that it may be effortful, delicate, or contingent on particular attentional control settings.

Acknowledgments This work was supported by a Discovery Grant to J.I. from the Natural Sciences and Engineering Research Council (NSERC) of Canada.

\section{References}

Broadbent, D.E. (1958). Perception and Communication. Toronto, ON: Pergamon Press, Ltd.

Carrasco, M. (2011). Visual attention: The past 25 years. Vision Research, 51, 1484-1525.

Cepeda, N. J., Cave, K. R., Bichot, N. P., \& Kim, M.-S. (1998). Spatial selection via feature-driven inhibition of distractor locations. Perception \& Psychophysics, 60(5), 727-746.

Chao, H.-F. (2010). Top-down attentional control for distractor locations: The benefit of precuing distractor locations on target localization and discrimination. Journal of Experimental Psychology: Human Perception \& Performance, 36(2), 303-316.

Cheal, M., \& Gregory, M. (1997). Evidence of limited capacity and noise reduction with single-element displays in the location-cuing paradigm. Journal of Experimental Psychology: Human Perception and Performance, 23(1), 51-71.

Chica, A. B., Klein, R. M., Rafal, R. D., \& Hopfinger, J. B. (2010). Endogenous saccade preparation does not produce inhibition of return: Failure to replicate Rafal, Calabresi, Brennan, \& Sciolto

\footnotetext{
${ }^{6}$ We thank another anonymous reviewer for raising this issue.
} 
(1989). Journal of Experimental Psychology: Human Perception and Performance, 36(5), 1193-1206.

Dosher, B. A., \& Lu, Z. L. (2000). Noise exclusion in spatial attention. Psychological Science, 11(2), 139-146.

Downing, P. E. (2000). Interactions between visual working memory and selective attention. Psychological Science, 11(6), 467-473.

Driver, J., Davis, G., Ricciardelli, P., Kidd, P., Maxwell, E., \& BaronCohen, S. (1999). Gaze perception triggers reflexive visuospatial orienting. Visual Cognition, 6(5), 509-540.

Egeth, H. E., \& Yantis, S. (1997). Visual attention: Control, representation, and time course. Annual Review of Psychology, 48(1), 269297.

Eriksen, C. W., \& Yeh, Y.-Y. (1985). Allocation of attention in the visual field. Journal of Experimental Psychology: Human Perception \& Performance, 11(5), 583-597.

Friesen, C. K., Ristic, J., \& Kingstone, A. (2004). Attentional effects of counterpredictive gaze and arrow cues. Journal of Experimental Psychology: Human Perception and Performance, 30(2), 319-329.

Giordano, A.M., McElree, B., \& Carrasco, M. (2009). On the automaticity and flexibility of covert attention: A speed-accuracy trade-off analysis. Journal of Vision, 9(3):30, 1-10. http://journalofvision. org/9/3/30/, doi:10.1167/9.3.30

Ivanoff, J., \& Saoud, W. (2009). Nonattentional effects of nonpredictive central cues. Attention, Perception, \& Psychophysics, 71(4), 872880

Klein, R. M., \& Hansen, E. (1990). Chronometric analysis of apparent spotlight failure in endogenous visual orienting. Journal of Experimental Psychology: Human Perception and Performance, 16(4), 790-801.

Klein, R. M., \& Hilchey, M. D. (2011). Oculomotor inhibition of return. The Oxford Handbook of Eye Movements, 471-492.

Lahav, A., Makovski, T., \& Tsal, Y. (2012). White bear everywhere: Exploring the boundaries of the attentional white bear phenomenon. Attention, Perception, \& Psychophysics, 74, 661-673.

Love, J., Selker, R., Marsman, M., Jamil, T., Dropmann, D., Verhagen, A. J., Ly, A., Gronau, Q. F., Smira, M., Epskamp, S., Matzke, D., Wild, A., Knight, P., Rouder, J. N., Morey, R. D., \& Wagenmakers, E.-J. (2016). JASP (Version 0.7.5.5) [Computer software].

Lu, Z. L., \& Dosher, B. A. (2000). Spatial attention: Different mechanisms for central and peripheral temporal precues? Journal of Experimental Psychology: Human Perception and Performance, 26(5), 1534.

Moher, J., \& Egeth, H. E. (2012). The ignoring paradox: Cueing distractor features leads first to selection, then to inhibition of tobe-ignored items. Attention, Perception, \& Psychophysics, 74(8), $1590-1605$

Munneke, J., van der Stigchel, S., \& Theeuwes, J. (2008). Cueing the location of a distractor: An inhibitory mechanism of spatial attention? Acta Psychologica, 129, 101-107.

Nickerson, R. S. (2000). Null hypothesis significance testing: A review of an old and continuing controversy. Psychological Methods, 5(2), 241-301.

Olivers, C. N. L., Meijer, F., \& Theeuwes, J. (2006). Feature-based memory-driven attentional capture: Visual working memory content affects visual attention. Journal of Experimental Psychology: Human Perception and Performance, 32(5), 1243-1265.

Posner, M. I. (1980). Orienting of attention. Quarterly Journal of Experimental Psychology, 32(1), 3-25.

Posner, M.I. (2014). Orienting of attention: Then and now. The Quarterly Journal of Experimental Psychology, doi:10.1080 /17470218.2014.937446.

Posner, M. I., \& Cohen, Y. (1984). Components of visual orienting. In H. Bouma \& D. G. Bouwhuis (Eds.), Attention and performance X: Control of language processes (pp. 531-556). Hillsdale, NJ: Erlbaum.
Posner, M. I., Cohen, Y., \& Rafal, R. (1982). Neural systems control of spatial orienting. Philosophical Transactions of the Royal Society B, 298, 187-198.

Posner, M. I., Snyder, C. R., \& Davidson, B. J. (1980). Attention and the detection of signals. Journal of Experimental Psychology: General, 109(2), 160-174.

Rafal, R. D., Calabresi, P. A., Brennan, C. W., \& Sciolto, T. K. (1989). Saccade preparation inhibits reorienting to recently attended locations. Journal of Experimental Psychology: Human Perception and Performance, 15(4), 673-685.

Rafal, R., \& Henik, A. (1994). The neurology of inhibition: Integrating controlled and automatic processes. In D. Dagenbach \& T. H. Carr (Eds.), Inhibitiory processes in attention, memory, and language (pp. 1-51). San Diego: Academic Press.

Riggio, L., \& Kirsner, K. (1997). The relationship between central cues and peripheral cues in covert visual orientation. Perception \& Psychophysics, 59(6), 885-899.

Ristic, J., Friesen, C. K., \& Kingstone, A. (2002). Are eyes special? It depends on how you look at it. Psychonomic Bulletin \& Review, 9(3), 507-513.

Ristic, J., \& Kingstone, A. (2006). Attention to arrows: pointing to a new direction. The Quarterly Journal of Experimental Psychology, 59(11), 1921-1930.

Ristic, J., \& Kingstone, A. (2012). A new form of human spatial attention: Automated symbolic orienting. Visual Cognition, 20(3), 244-264.

Rouder, J. N., Morey, R. D., Speckman, P. L., \& Province, J. M. (2012). Default Bayes factors for ANOVA designs. Journal of Mathematical Psychology, 56, 356-374.

Rouder, J. N., Speckaman, P. L., Sun, D., Morey, R. D., \& Iverson, G. (2009). Bayesian $t$ tests for accepting and rejecting the null hypotheisis. Psychonomic Bulletin \& Review, 16(2), 225-237.

Slotnick, S. D., Hopfinger, J. B., Klein, S. A., \& Sutter, E. E. (2002). Darkness beyond the light: attentional inhibition surrounding the classic spotlight. Neuroreport, 13(6), 773-778.

Slotnick, S. D., Schwarzbach, J., \& Yantis, S. (2003). Attentional inhibition of visual processing in human striate and extrastriate cortex. Neuroimage, 19(4), 1602-1611.

Soto, D., Heinke, D., Humphreys, G. W., \& Blanco, M. J. (2005). Early, involuntary top-down guidance of attention from working memory. Journal of Experimental Psychology: Human Perception and Performance, 31(2), 248-261.

Summerfield, C., \& Egner, T. (2009). Expectation (and attention) in visual cognition. Trends in Cognitive Science, 13(9), 403-409.

Tipper, S. P. (2001). Does negative priming reflect inhibitory mechanisms? A review and integration of conflicting views. The Quarterly Journal of Experimental Psychology: Section A, 54(2), 321-343.

Taylor, T. L., \& Klein, R. M. (2000). Visual and motor effects in inhibition of return. Journal of Experimental Psychology: Human Perception and Performance, 26(5), 1639-1656.

Tsal, Y., \& Makovski, T. (2006). The attentional white bear phenomenon: The mandatory allocation of attention to expected distractor locations. Journal of Experimental Psychology: Human Perception \& Performance, 32(2), 351-363.

Verbruggen, F., \& Logan, G. D. (2008). Response inhibition in the stopsignal paradigm. Trends in Cognitive Sciences, 12(11), 418-424.

Vossel, S., Thiel, C. M., \& Fink, G. R. (2006). Cue validity modulates the neural correlates of covert endogenous orienting of attention in parietal and frontal cortex. NeuroImage, 32(3), 1257-1264.

Wagenmakers, E. J. (2007). A practical solution to the pervasive problems of $p$ values. Psychonomic Bulletin \& Review, 14(5), 779-804.

Wagenmakers, E. J., Lodewyckx, T., Kuriyal, H., \& Grasman, R. (2010). Bayesian hypothesis testing for psychologists: a tutorial on the Savage-Dickey method. Cognitive Psychology, 60, 158-189. 
Warner, C. B., Juola, J. F., \& Koshino, H. (1990). Voluntary allocation versus automatic capture of visual attention. Perception \& Psychophysics, 48(3), 243-251.

Wegner, D. M. (1994). Ironic processes of mental control. Psychological Review, 101, 34-52.

Wegner, D. M., Ansfield, M., \& Pilloff, D. (1998). The putt and the pendulum: ironic effects of the mental control of action. Psychological Science, 9(3), 196-199.
Wegner, D. M., Schneider, D. J., Carter, S. R., \& White, T. L. (1987). Paradoxical effects of thought suppression. Journal of Personality and Social Psychology, 53(1), 5-13.

Yantis, S., \& Jonides, J. (1984). Abrupt visual onsets and selective attention: evidence from visual search. Journal of Experimental Psychology: Human Perception \& Performance, 10, 601-21.

Yantis, S., \& Jonides, J. (1990). Abrupt visual onsets and selective attention: Voluntary versus automatic allocation. Journal of Experimental Psychology: Human Perception \& Performance, 16(1), 121-134. 